\title{
Application of the wavelet transform and the enhanced Fourier spectrum in the impact echo test
}

\author{
Po-Liang Yeh, Pei-Ling Liu* \\ Institute of Applied Mechanics, National Taiwan University, Taipei 106, Taiwan \\ Received 14 May 2007; received in revised form 15 December 2007; accepted 2 January 2008 \\ Available online 12 January 2008
}

\begin{abstract}
The objective of this study is to develop a reliable and effective method to analyze the signal of the impact echo test. The impact echo test is a nondestructive testing technique for civil structures. In the test, the surface response of the target structure due to an impact is measured. Then, the Fourier transform is adopted to transform the signal from the time domain to the frequency domain. Owing to the multiple reflections induced by cracks, voids, or other interfaces, peaks will form in the Fourier spectrum. The frequencies of the peaks can then be used to determine the size of the structure or the location of the defect.

Several difficulties are encountered when applying the Fourier transform to impact echo data. Because the impact echo data are nonstationary and contains multiple reflections, ripples and multiple peaks appear in the Fourier spectrum, which may mislead the follow-up diagnosis. Furthermore, the existence of the high-energy surface wave and structural vibrations often complicates the spectrum and makes the data interpretation even more difficult.

To overcome these difficulties, this research adopts the wavelet transform in the analysis of impact echo data. Theoretically, the wavelet transform can avoid ripple and multiple-peak phenomena. Furthermore, the frequency range and time span of surface wave can be observed in the wavelet scalogram. However, the spectral resolution of the wavelet marginal spectrum is inferior to that of the Fourier transform. Therefore, two approaches are proposed in this paper. One is to combine the Fourier spectrum and the wavelet marginal spectrum to determine the precise location of the echo peak. The other is to take the product of the two spectra to establish the enhanced Fourier spectrum. As such, the interference in the Fourier spectrum is suppressed while the peak is enhanced. Numerical and experimental tests were performed to verify the effectiveness and reliability of the proposed approaches.
\end{abstract}

(C) 2008 Elsevier Ltd. All rights reserved.

Keywords: Impact echo test; Fourier transform; Wavelet transform; Nondestructive test; Enhanced Fourier spectrum

\section{Introduction}

The impact echo method is widely applied in the nondestructive testing of concrete structures. In its early stages of development, the impact echo method was applied primarily to detect internal flaws in concrete plate $[1,2]$. Later it was used for the inspection of the flaws in rod structures [3], flaws in concrete panels [4], and the corrosion damage of rebars in concrete [5].

\footnotetext{
*Corresponding author. Tel.: + 886233665622 ; fax: + 886233665623 .

E-mail address: peiling@iam.ntu.edu.tw (P.-L. Liu).
}

In the impact echo test, a steel ball or a hammer is used to knock at the surface of the specimen. Consequently, stress waves will be generated and propagate in the structure, including the longitudinal wave, the transverse wave, and the surface wave. The surface wave propagates along the surface, while the transverse wave and the longitudinal wave propagate into the interior of the structure. As the bulk wave encounters an interface such as crack or boundary, it will be reflected, refracted, or diffracted. A transducer is placed near the impact point to measure the response of the structure. Then, the received signals are recorded for data analysis.

Since the received signals contain the surface wave and the reflected or diffracted bulk wave, it is difficult to 
extract useful information directly from the raw data. Therefore, the conventional impact echo method applies the Fourier transform (FT) to the time signal. The idea is as follows: When a wave propagates in a structure, it reflects as it encounters an interface. Then the reflected wave rebounds back to the surface and reflect again into the interior of the structure. The process repeats and multiple reflections occur between the surface and the interface until the wave fades out. The echo of the wave will form a peak in the Fourier spectrum, and its frequency $f$ is related to the depth of the interface $D$ by the following equation [1]:

$f=\frac{C_{\mathrm{p}}}{2 D}$

where $C_{\mathrm{p}}$ is the velocity of the longitudinal wave. Thus, by locating the peaks in the Fourier spectrum, the size of the structure or the depth of an internal crack can be determined.

The FT has been adopted to detect the size or flaws of concrete structures successfully in numerous cases. However, the interpretation of Fourier spectrum is by no means easy because it contains a lot of interference which is intrinsic in the impact echo spectrum.

The FT theory is built on the assumption of a stationary signal. However, the impact response of a structure is not stationary, and its frequency content varies with time. Hence, interference appears in the spectrum, such as ripples and multiple peaks. Furthermore, the existence of the highenergy surface wave and structural vibrations often complicates the spectrum and makes the data interpretation even more difficult.

To deal with the aforementioned difficulties, several time-frequency analyses were proposed for use in the impact echo test, for example, the wavelet transform (WT), the short-time Fourier transform (STFT), and the Hilbert-Huang transform (HHT). Abraham et al. [6] proposed a windowed FT for the detection of voids in a concrete block. Kim and Kim [7] used WT to find the position of an opening crack in a rod-liked structure. Chiang and Cheng [8] employed WT to inspect steel tubes and PVC tubes. Shokouhi et al. [9] used STFT and WT in the size detection of concrete block. Algernon and Wiggenhauser [10] compared the performance of STFT, WT and HHT in the measurement of structural dimension. Different from the aforementioned papers, in which data interpretation was performed in the time-frequency plane, the marginal spectra were used to determine the depth of structural members. The time-frequency analyses were also adopted in the evaluation of structures using other nondestructive tests [11-16].

This paper studies in depth the application of WT in the impact echo test of concrete structures with internal cracks. The results of FT and WT will be compared and discussed. Based on the investigation, a better data processing approach for the impact echo test will be devised.

\section{Signal analysis}

\subsection{Fourier transform}

The FT of a signal $x(t)$ and its inverse transform are as follows:

$X(f)=\int_{-\infty}^{+\infty} x(t) \mathrm{e}^{-2 \mathrm{i} \pi f t} \mathrm{~d} t$

$x(t)=\int_{-\infty}^{+\infty} X(f) \mathrm{e}^{2 \mathrm{i} \pi f t} \mathrm{~d} f$.

The above equations imply the signal $x(t)$ is expanded in terms of the harmonic function $\mathrm{e}^{2 \mathrm{i} \pi f t}$ from $t=-\infty$ to $+\infty$, and $X(f)$ represents the magnitude of the component with frequency $f$. It is seen that the signal is transformed completely from the time domain to the frequency domain. Hence, $X(f)$ contains no information on the temporal variation of the frequency content.

FT has two drawbacks when applied to impact echo data. Firstly, FT expands signals by stationary harmonic functions, but the impact echo signal is non-stationary. Hence, extra components are needed to approximate the sudden changes in the signal. These additional frequency components incur ripples in the spectrum. This is known as Gibb's phenomenon.

Secondly, FT produces multiple peaks in the spectrum due to the time shifting property [17]. The time shifting property of FT is as follows:

$\operatorname{FT}\left[x\left(t-t_{0}\right)\right]=\mathrm{e}^{-\mathrm{i} 2 \pi f t_{0}} X(f)$,

where FT[ $\cdot]$ represents the FT operator. In the impact echo test, the stress wave propagates back and forth between the surface and interface. When the stress wave propagates back to the surface, a new source is generated with a time lag of $t_{0}=D / 2 C_{\mathrm{p}}$. If the Fourier spectrum of the wave due to the first source is $X(f)$, the Fourier spectrum of the wave due to the new source is approximately equal to $\mathrm{e}^{-\mathrm{i} 2 \pi f t_{0}} X(f)$. The same argument can be extended to the multiple reflected waves. The superposition of these spectra would result in repeated peaks in the spectrum at a frequency interval of $1 / t_{0}$.

The aforementioned interferences may jumble the real echo peaks and jeopardize the interpretation of impact echo data.

\subsection{Wavelet transform}

The FT cannot provide the frequency content in a certain time interval. To remedy the situation, the most direct approach is to apply FT within a limited time interval. As such, the Fourier spectrum will reflect the frequency content of the signal in that time interval. Then, one can shift the time window and obtain the frequency content of the signal for different time intervals. This is the basic idea of the STFT [18]. 
The authors have studied the STFT in the impact echo test [19]. It is found that although the STFT can get rid of the ripples, multiple peaks remain. Furthermore, the frequency resolution of STFT is not satisfactory.

Similar to the STFT, the WT is also a time-frequency analysis. The WT of a signal $x(t)$ is as follows [20]:

$W(a, \tau)=\frac{1}{\sqrt{a}} \int_{-\infty}^{+\infty} x(t) \Psi\left(\frac{t-\tau}{a}\right) \mathrm{d} t$,

where $\psi(t)$ is the mother wavelet function, $\tau$ is the translation parameter, and $a$ is the dilation parameter. The factor $1 / \sqrt{a}$ is a normalization parameter that makes the energy of each wavelet unitary.

Researchers have proposed several wavelet functions $[21,22]$. Consider the Morlet wavelet function for example. It has the following form:

$\Psi(t)=\mathrm{e}^{-t^{2} / 2} \mathrm{e}^{\mathrm{i} C t}$,

where $C$ is the frequency center. Notice that $\psi$ is a sinusoidal function modulated by the function $\mathrm{e}^{-t^{2} / 2}$. Since $\mathrm{e}^{-t^{2} / 2}$ decays exponentially with $t^{2}$, it serves as a time window. Substituting Eq. (6) into Eq. (5) and comparing it with Eq. (2), one can see that WT is almost identical to FT except the use of the time window function for the time-frequency analysis. Other wavelet functions take different forms. However, they share the same property that the amplitude nearly vanishes outside a bounded range. In other words, their amplitudes are noticeable only within a time window.

For use in the impact echo test, it is convenient to express the scale $a$ in WT in terms of frequency $f$ (or pseudo-frequency). For the Morlet wavelet function, the relation between $a$ and $f$ is [23]

$f=\frac{C}{2 \pi a \Delta t}$,

where $\Delta t$ is the sampling period. Using such relation, one can express the WT of a signal as a function of $f$ and $\tau$, that is, $W(f, \tau)$.

The density plot of $|W(a, \tau)|^{2}$ or $|W(f, \tau)|^{2}$ is called the scalogram. It represents the energy distribution of the signal in time-frequency domain. Hence, the temporal variation of the frequency content of a signal can be observed directly in the scalogram. One can integrate the scalogram with respect to time to obtain the marginal spectrum of WT [18]:

$Y(f)=\int_{0}^{+\infty}|W(f, \tau)|^{2} \mathrm{~d} \tau$.

Similar to the Fourier spectrum, the wavelet marginal spectrum provides the frequency content of a signal in the entire time domain.

Since WT is applicable to non-stationary data, no ripples are induced in the marginal spectrum. Moreover, WT has a different time shifting relation than FT:

$\mathrm{WT}\left[x\left(t-t_{0}\right)\right]=W\left(f, \tau-t_{0}\right)$, where WT[ · ] represents the WT operator. Therefore, there is no evident ground for the appearance of multiple peaks.

WT has another advantage. It is known that the surface wave contains no information about the internal defect or the size of the structure. However, its energy is high and it forms a rough hump in the spectrum. If the echo is weak, it may become less noticeable. Using the scalogram, the time span of the surface wave can be roughly determined. Hence, one can try to locate the echo peak after the surface wave diminishes.

WT also has a feature that is attractive to the impact echo test: the frequency resolutions of the scalogram and marginal spectrum increase as frequency decreases. This is because as the width of the time window increases, the dominant frequency of the wavelet function decreases and the frequency resolution improves. Consequently, WT has a better frequency resolution in the low-frequency range than in the high frequency range.

This feature happens to satisfy the need of the impact echo test. Recall that the depth of the interface is inversely proportional to the peak frequency. Suppose the error of peak frequency is $\Delta f$. According to Eq. (1), the induced error in the interface depth can be written as follows:

$$
\begin{aligned}
\Delta D & =\left[\frac{1}{1+(\Delta f / f)}-1\right] D=-\frac{\Delta f}{f} D+\frac{1}{2}\left(\frac{\Delta f}{f}\right)^{2} D-\cdots \\
& =-\frac{C_{\mathrm{p}}}{2 f^{2}} \Delta f+\cdots
\end{aligned}
$$

If the higher order terms in Eq. (10) are neglected, the depth error $\Delta D$ is proportional to $\Delta f / f^{2}$ and the relative error $\Delta D / D$ is proportional to $\Delta f / f$. If one wants to keep $\Delta D$ or $\Delta D / D$ constant within a frequency range, $\Delta f$ must decrease with $f$. This is not likely to occur in FT and STFT, because the frequency resolutions in both analyses are constant. On the other hand, WT has a better frequency resolution in the low-frequency range. Hence, one could expect that $\Delta f$ decreases with $f$. Therefore, the accuracy of the detected depth in WT is more stable than in FT and STFT as the peak frequency varies.

\subsection{Enhanced Fourier spectrum and combined spectra}

Although WT has several advantages over FT, it never surpasses FT in frequency resolution. This can be explained by the uncertainty principle of the timefrequency analysis:

$\sigma_{t} \sigma_{f} \geqslant \frac{1}{4 \pi}$,

where $\sigma_{t}$ and $\sigma_{f}$ denote the resolution in the time and frequency domain, respectively. The principle states that the optimal time resolution and frequency resolution cannot be achieved simultaneously. Since FT does not provide temporal information, it has the worst time resolution but best frequency resolution. As a result, the echo peak in the wavelet marginal spectrum is not as sharp 
as in the Fourier spectrum. Very often one only finds a hump in the marginal spectrum. That makes the peak frequency difficult to pinpoint.

In order to incorporate the advantages of both FT and WT, an enhanced Fourier spectrum is proposed as follows:

$E(f)=X(f) Y(f)$,

where $E(f)$ is the enhanced Fourier spectrum, $Y(f)$ is the wavelet marginal spectrum.

The wavelet marginal spectrum can be considered as a band-pass filter. Multiplying $X(f)$ with $Y(f)$ amounts to applying the band-pass filter $Y(f)$ to $X(f)$. It is seen that the frequency components in $X(f)$ around the peak of $Y(f)$ are magnified, while the frequency components away from the peak are held back, as shown in Fig. 1. As such, the ripples and multiple peaks in the Fourier spectrum are suppressed. On top of that, the echo peak in $X(f)$ is further enhanced because it coincides with the peak of $Y(f)$. Consequently,
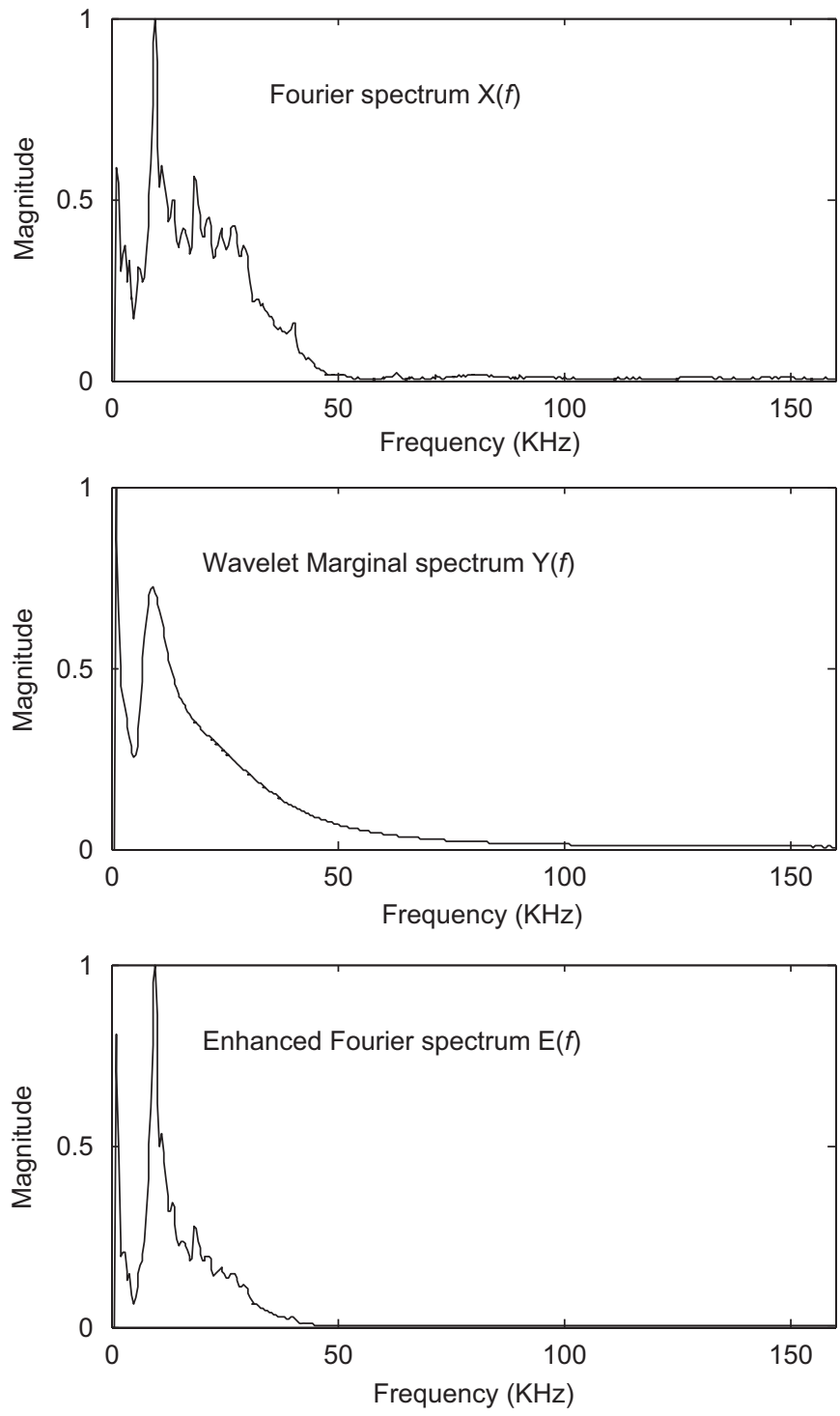

Fig. 1. Comparison of the Fourier spectrum, wavelet marginal spectrum, and the enhanced Fourier spectrum. there is no ambiguity in locating the echo peak in the enhanced spectrum and the peak in $E(f)$ is even sharper than in $X(f)$.

There is yet another way to pinpoint the echo peak correctly and precisely other than using the enhanced Fourier spectrum. One may combine the Fourier spectrum and the wavelet marginal spectrum in the same figure. Since the wavelet marginal spectrum contains no ripples and multiple peaks, one can easily use it to find the hump formed by the interface echo. Although the hump may be smooth, it will provide the approximate location of the peak. Then, one may zoom in the figure and use the Fourier spectrum to determine the location of the peak precisely.

In the following, numerical examples and model tests will be presented to compare the aforementioned signal processing methods.

\section{Numerical simulation}

This study adopted the finite element code LS-Dyna970 [24] to simulate the response of concrete specimens due to the impact of a steel ball. It is also used to perform modal analysis to find the natural frequencies of the specimens.

Two concrete specimens with internal cracks are considered in the numerical examples. The mass density, Young's modulus, Poisson's ratio, and the longitudinal wave speed of the concrete are $2300 \mathrm{~kg} / \mathrm{m}^{3}, 33.1 \mathrm{GPa}, 0.2$, and $4000 \mathrm{~m} / \mathrm{s}$, respectively. The dimensions of the numerical models are identically $0.8 \mathrm{~m}(\mathrm{~L}) \times 0.8 \mathrm{~m}(\mathrm{~W}) \times 0.2 \mathrm{~m}$ $(\mathrm{H})$. Absorbing boundary conditions are applied on the four sides of the models. This prevents waves reflecting from the boundaries and makes it easier to demonstrate the proposed method. Therefore, the numerical models, in fact, simulate large plates rather than concrete blocks.

A time-varying pressure was applied to the surface to simulate the impact of a steel ball with diameter $d=6 \mathrm{~mm}$. According to Goldsmith [25], the pressure can be approximated by a half sine function with a contact time $t_{\mathrm{c}}=25 \mu \mathrm{s}$. The maximum frequency of the excited stress wave is about $50 \mathrm{kHz}$ [26], and the minimum wavelength is about $8 \mathrm{~cm}$.

Three-dimensional solid elements were used in the simulation. Generally, the mesh size is set as $1 / 8-1 / 10$ of the wavelength in the transient wave analysis. Since the minimum wavelength is $8 \mathrm{~cm}$ in the numerical examples, the concrete specimens were divided into cubic elements with side length $1 \mathrm{~cm}$.

It was assumed that the receiver was located at $4 \mathrm{~cm}$ from the impact source. The total simulation time was $3 \mathrm{~ms}$, and the time increment was $3 \mathrm{~ms} / 1024=2.93 \mu \mathrm{s}$.

\subsection{Numerical model 1}

Consider a specimen containing a $0.32 \mathrm{~m}(\mathrm{~L}) \times 0.32 \mathrm{~m}$ $(\mathrm{W}) \times 0.01 \mathrm{~m}(\mathrm{H})$ horizontal crack, which is $0.12 \mathrm{~m}$ beneath 


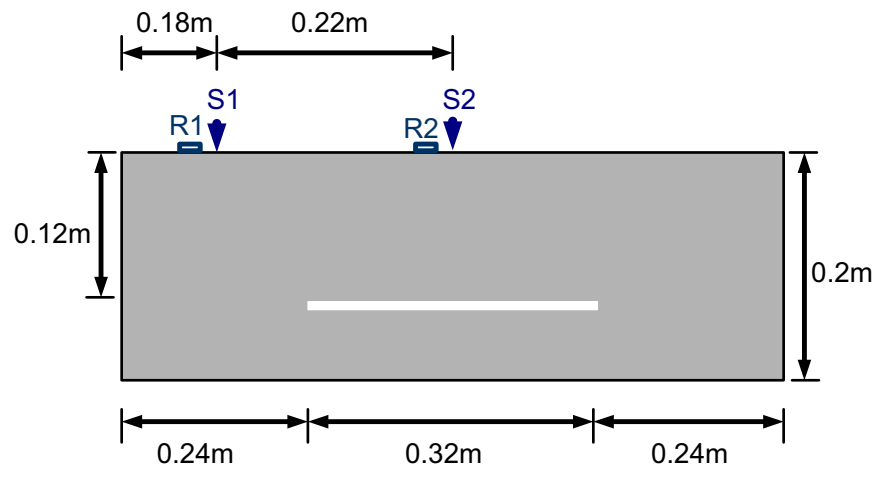

Fig. 2. Model 1.

the surface. The side view of specimen is illustrated in Fig. 2.

Two numerical tests were performed. In test 1 , the impact source is $0.18 \mathrm{~m}$ away from the model edge; while in test 2, the impact source is $0.40 \mathrm{~m}$ away from the model edge, as shown in Fig. 2. In the figure, " $\mathrm{S} n$ " and " $\mathrm{R} n$ " denote the locations of the source and receiver, respectively, where $n$ denotes the test number.

Fig. 3(a) shows the time signal of the test. Theoretically, the direct longitudinal wave should arrive at the receiver first. However, it is very weak and not observable in Fig. 3(a). The direct longitudinal wave is followed by the strong surface wave. Then, the signal fluctuates and gradually fades out. One cannot obtain much information by inspecting the time signal directly.

As mentioned previously, the existence of the surface wave may complicate the Fourier spectrum. To illustrate this, the surface wave is removed from the signal on purpose, as shown in Fig. 3(b). The Fourier spectra of the original and modified signals are shown in Fig. 3(c) and (d), respectively. The difference between the original and modified spectra is shown in Fig. 3(e). One can find a rough hump in the frequency range of $10-40 \mathrm{kHz}$. This is caused by the surface wave. Although the height of the hump is about the same as the echo peak in Fig. 3(d), there is no difficulty in identifying the echo peak in this case. However, the energy of the crack echoes decreases, as the crack gets smaller or deeper. Apparently, the echo peak may become difficult to detect in some situations.

Fig. 4 shows the scalograms and WT marginal spectra of this example using three different wavelet functions, namely, the Morlet wavelet, the Symlet order 8 wavelet, and the Mexican hat wavelet $[20,23]$. In each scalogram, there is a vertical bright zone near the time origin, which is caused by the strong surface wave. Obviously, the scalogram reveals the frequency range and time span of the surface wave.

There is a horizontal bright band adjacent to the time axis spanning almost the entire time axis. It means that the signal decays slowly. Compared with the results of modal analysis, this band corresponds to first vibration mode of the model.
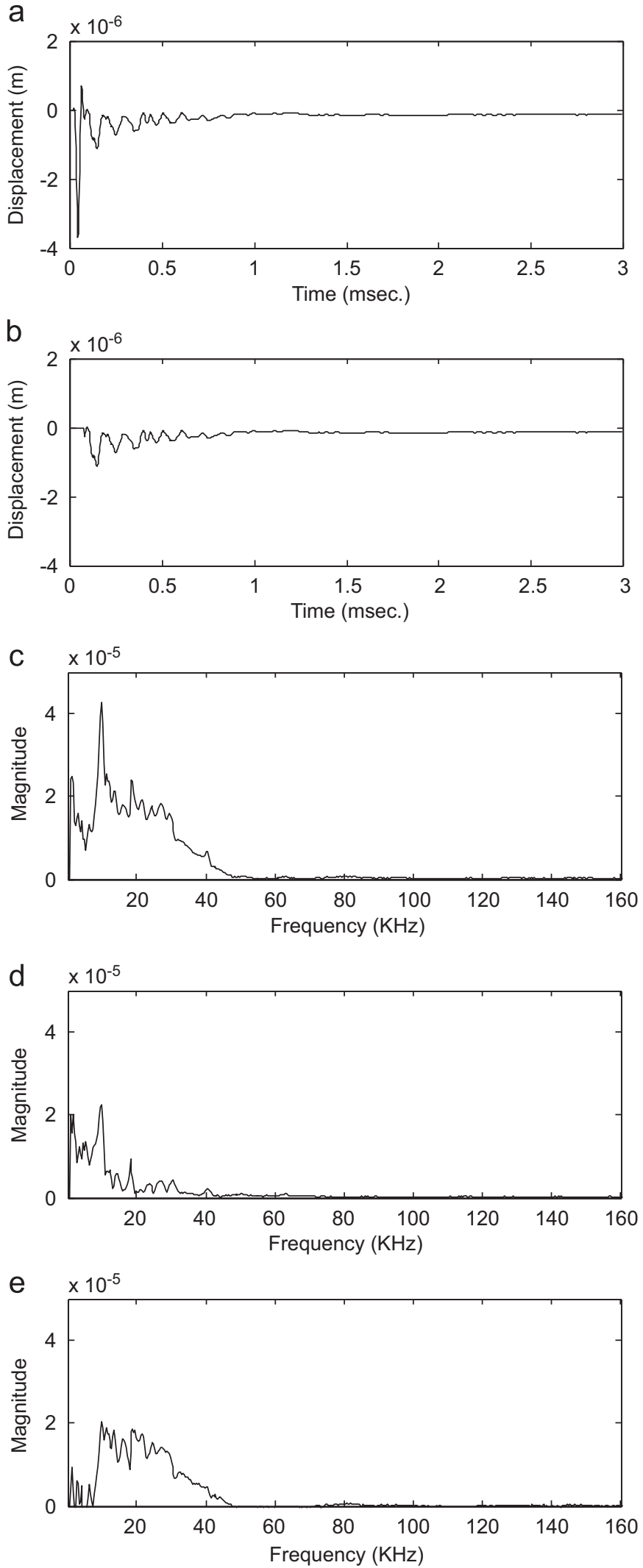

Fig. 3. Time signals and spectra of test 1 , numerical model 1. (a) Original time signal; (b) time signal with surface removed; (c) Fourier spectrum of the original time signal; (d) Fourier spectrum of time signal with surface wave removed and (e) Fourier spectrum of the surface wave. 
a

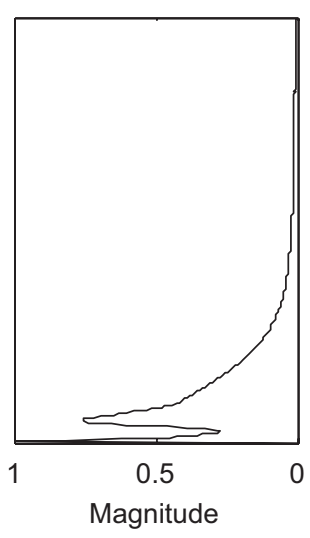

b

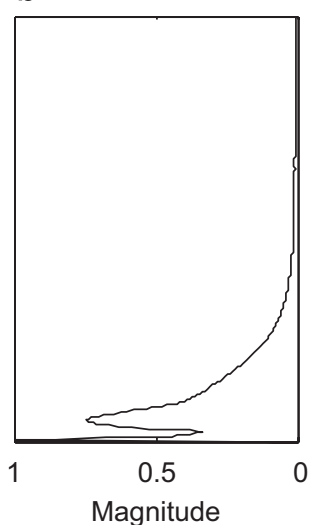

。

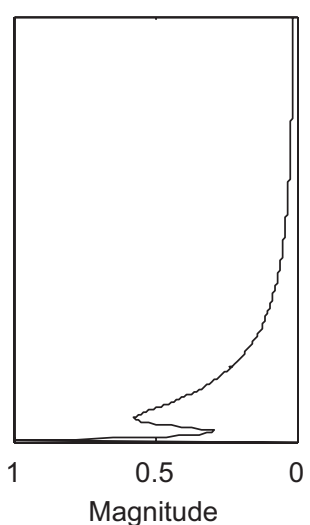

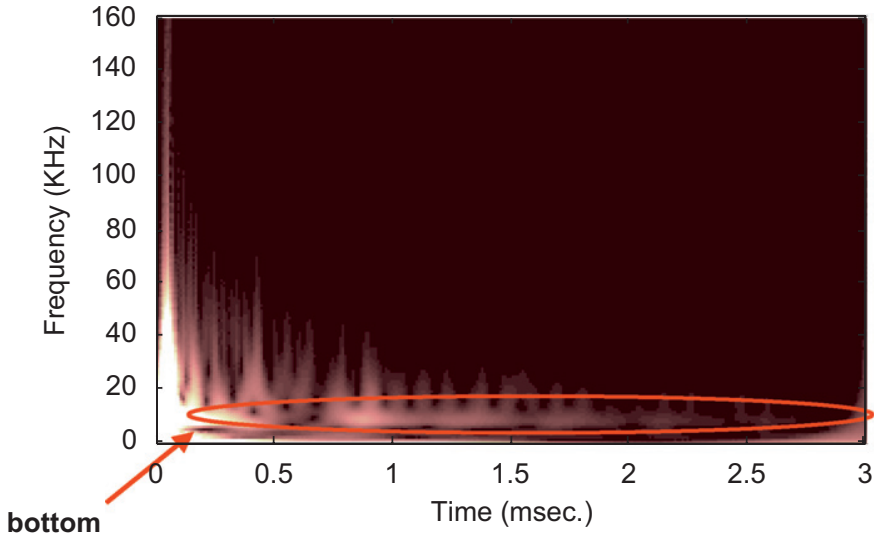
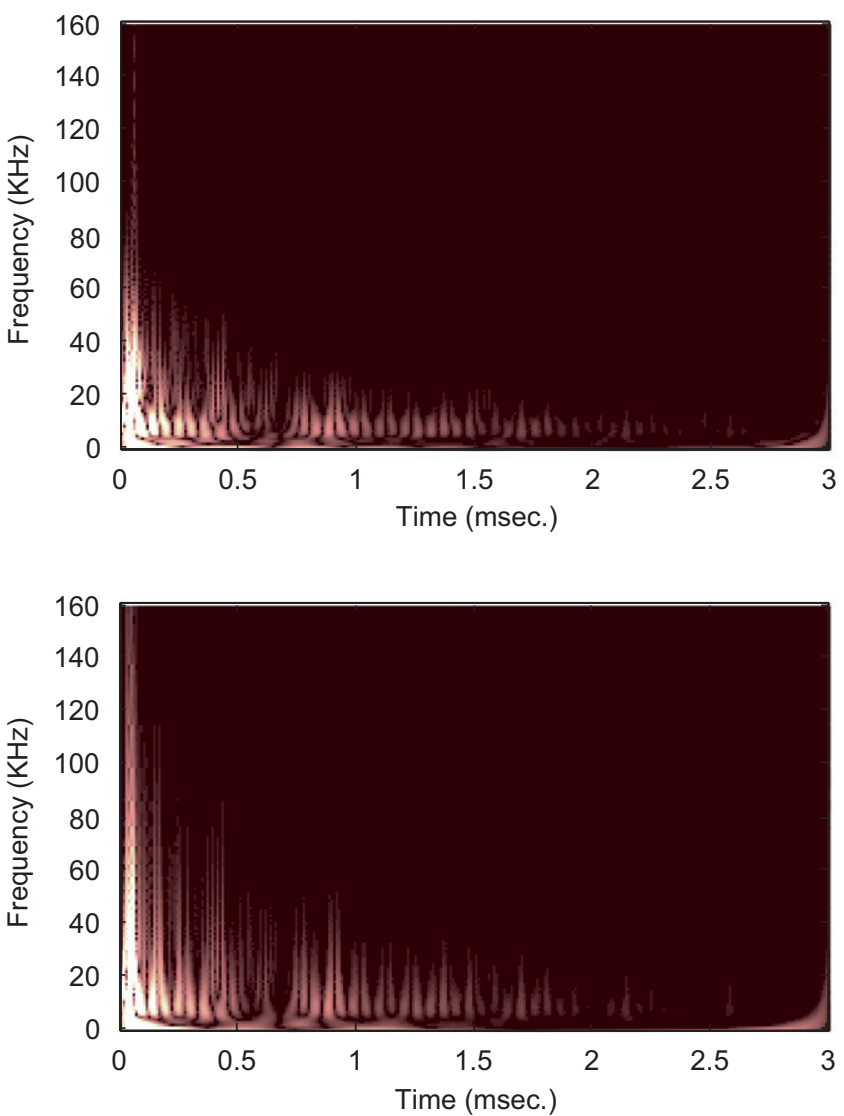

Fig. 4. Influence of wavelet function on the scalogram and wavelet marginal spectrum of test 1, numerical model 1. (a) Morlet wavelet; (b) Symlet wavelet and (c) Mexican hat wavelet.

There is another horizontal bright band at approximately $10 \mathrm{kHz}$. According to Eq. (1), this is apparently caused by the multiple reflections between the top and bottom surfaces of the concrete specimen. This bright band is not as clear as the vertical bright zone of the surface wave. However, it is easy to distinguish them because they appear in different areas in the scalogram. This is certainly an advantage of the scalogram over the Fourier spectrum.

Comparing these figures, one can see that the horizontal bright band in the Morlet scalogram is the clearest among the three. The echo peak in the Morlet marginal spectrum is also the sharpest. In other words, the Morlet wavelet yields the best frequency resolution. Since the frequency resolution controls the accuracy of the impact echo method, the Morlet wavelet is adopted in this paper.

Although the Morlet wavelet is the most widely used wavelet in the literature, there is no guarantee that it always yields the best result. One should try out different wavelets to find the best choice in each application.

Fig. 5 shows the Morlet marginal spectra of test 1 for $C=0.5 \pi-3.0 \pi$. It is seen that the frequency resolution of the wavelet marginal spectrum increases with $C$. However, 

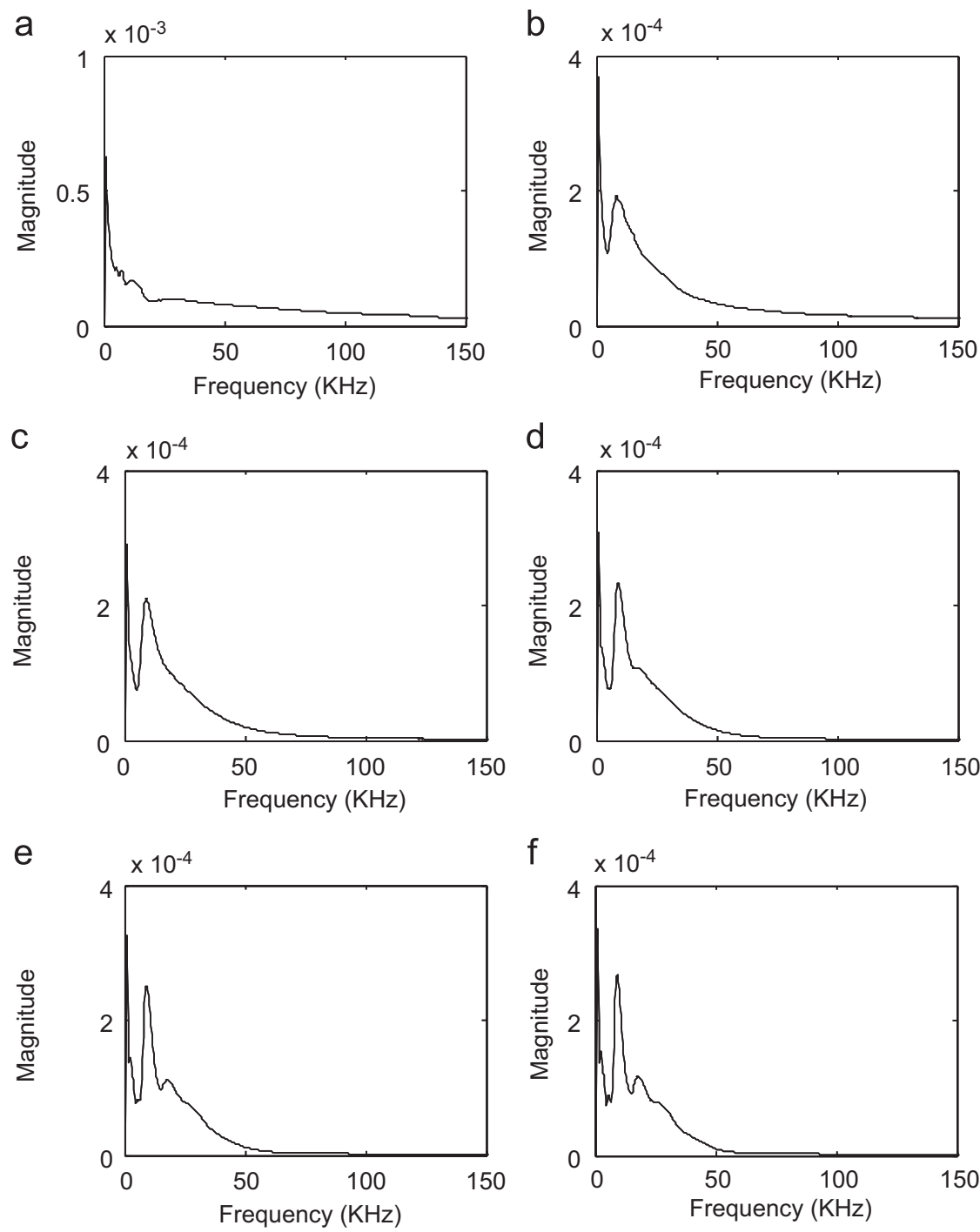

Fig. 5. Influence of the frequency center $C$ on the wavelet marginal spectrum of test 1 , numerical model 1 . (a) $C=0.5 \pi$; (b) $C=1.0 \pi$; (c) $C=1.5 \pi$; (d) $C=2.0 \pi$; (e) $C=2.5 \pi$ and (f) $C=3.0 \pi$.

the spectrum becomes jagged as $C$ increases. The $C$ value should be chosen such that it is large enough to provide good frequency resolution but not too large to avoid a jagged spectrum. In this case, the frequency of the peak frequency remains the same while interference starts to appear as $C$ exceeds $1.5 \pi$. Therefore, $C=1.5 \pi$ is adopted in this case. The same approach is used to determine the $C$ values in other cases.

Fig. 6 shows the Fourier spectrum, the wavelet marginal spectrum and the enhanced Fourier spectrum of the test signal. The highest peak in the Fourier spectrum is located at $9.78 \mathrm{kHz}$, which yields a bottom depth of $0.204 \mathrm{~m}$. The second highest peak is located at $1.35 \mathrm{kHz}$, which is very close to the fundamental natural frequency of the specimen obtained by modal analysis. Hence, it should come from the modal vibration of the specimen.

There are also peaks at the multiples of $10 \mathrm{kHz}$, e.g., $20,30 \mathrm{kHz}$, and so on. The Fourier spectrum also contains a lot of ripples. The existence of the multiple peaks and ripples makes the interpretation of the spectrum difficult.

On the other hand, the wavelet marginal spectrum is smooth. The peak at $1.35 \mathrm{kHz}$ still exists, as expected. The highest peak in the marginal spectrum, located at about $10 \mathrm{kHz}$, comes from the bottom echo.

The enhanced Fourier spectrum, the product of the Fourier spectrum and the wavelet marginal spectrum, is shown in Fig. 6(b). It is seen that it still contains ripples and multiple peaks. However, such interferences are suppressed to a reasonable extent. Clearly, it is easy to identify the peak due to the bottom echo. The frequency of the bottom echo in the enhanced Fourier spectrum is the same as in the Fourier spectrum.

Fig. 7 shows the results of test 2 . Again, the frequency range and time span of the surface wave can be clearly identified in the scalogram. Since there is a crack under the impact point, one can find a bright band at about $16 \mathrm{kHz}$ spanning the entire time axis. There are two other 
a

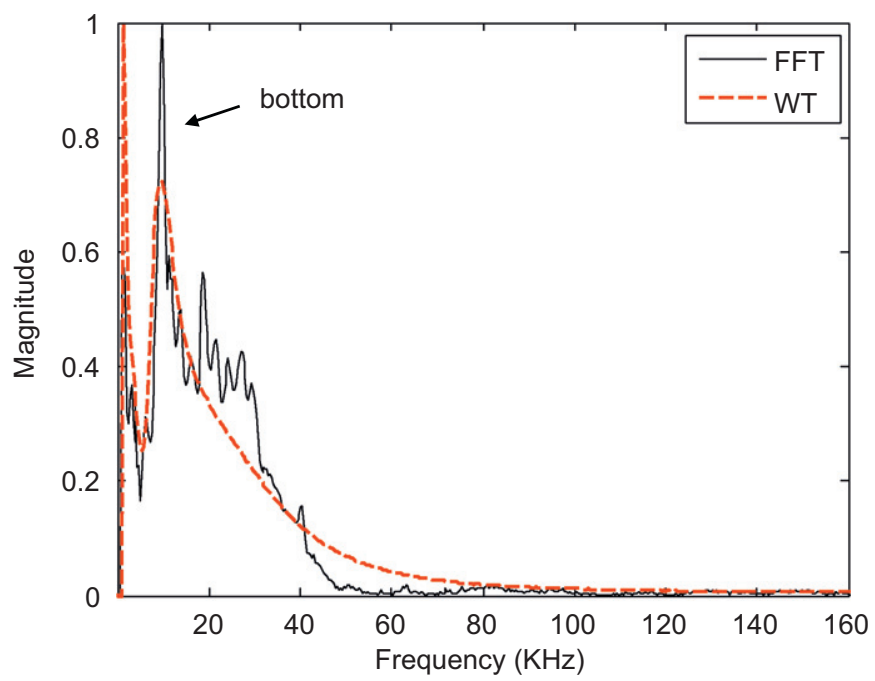

b

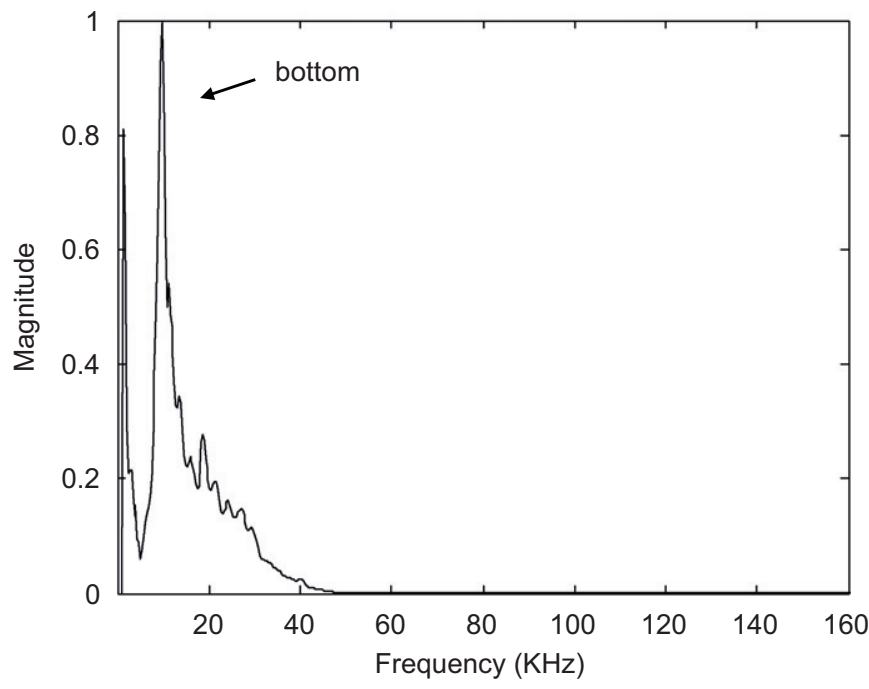

Fig. 6. (a) Fourier and wavelet marginal spectrum and (b) enhanced Fourier spectrum of test 1 , numerical model 1 .

horizontal bright bands, also spanning the entire time axis. Compared with the results of modal analysis, these bands correspond to two vibration modes with frequencies 2.36 and $7.09 \mathrm{kHz}$.

Similar to the first test, the inherent interferences still exist in the Fourier spectrum and the wavelet marginal spectrum is smoother than Fourier spectrum. There are peaks in all the spectra. The first two correspond to modal vibrations, and the third one comes from the crack echo.

Comparing the peaks in the wavelet marginal spectrum, one can see that the peak gets smoother as the frequency increases. Examining the horizontal bright bands in the scalogram, one can also see that the height of the band increases with frequency. Both phenomena illustrate the feature of WT that its frequency resolution decreases as the frequency increases.

In this case, the interferences in the Fourier spectrum are not as obvious as in test 1 . One does not have to resort to the enhanced Fourier spectrum or the combined spectra to detect the crack depth. Nevertheless, the enhanced Fourier spectrum is still easier to interpret among the three spectra. The crack frequency of the enhanced Fourier spectrum is $16.6 \mathrm{kHz}$, yielding a crack depth of $0.121 \mathrm{~m}$.

\subsection{Numerical model 2}

Consider again a specimen containing a $0.32 \mathrm{~m}$ $(\mathrm{L}) \times 0.32 \mathrm{~m}(\mathrm{~W}) \times 0.01 \mathrm{~m}(\mathrm{H})$ horizontal crack. In this case, the depth of the crack is $0.06 \mathrm{~m}$ beneath the surface, as shown in Fig. 8. An impact was applied on the surface of the concrete specimen right on top the crack, and the receiver was located at $0.04 \mathrm{~m}$ from the impact source. The locations of the source and receiver are designated as " $\mathrm{S}$ " and "R", respectively, in Fig. 8.

This example is presented here because the response of the concrete specimen with a shallow crack is dissimilar to that with a deep crack. Usually, it is more difficult to detect a shallow crack in the concrete using the impact echo test.

Fig. 9 shows the test results of model 2. Comparing the time responses in Fig. 9(a) with Fig. 7(a), one can see that the behavior of this specimen is quite different from that of model 1, although both tests were performed right on top of a crack. In Fig. 7(a), one can see multiple reflections riding on the low-frequency vibration throughout the time axis. In Fig. 9(a), the high-frequency multiple reflections only appear for a short period of time. The low-frequency vibration, on the other hand, is very strong and damps out slowly. The strong, continual vibration is a typical phenomenon if the impact echo test is performed on top of a shallow crack. This is because the impact would induce the flexural vibration of the thin plate between the top surface and the crack.

The phenomenon observed in the time signal reveals in the scalogram, as shown in Fig. 9(b). One can find a dominant bright band at the bottom of the scalogram, corresponding to the flexural vibration. There is another low-frequency bright band, also corresponding to a modal vibration but much weaker than then flexural vibration. The light band near $30 \mathrm{kHz}$ comes from crack echo. Although it is still observable, it only lasts for a short period of time.

Since the flexural vibration absorbs most of the energy, the echo peak is very small and hard to detect in the Fourier and wavelet marginal spectra. Therefore, the scales of the spectra need to be adjusted.

Comparing wavelet marginal spectra in Figs. 6(a), 7(c), and $9(\mathrm{c})$, one can see that the peaks corresponding to the echoes of the $0.20 \mathrm{~m}$ deep bottom, the $0.12 \mathrm{~m}$ deep crack, and the $0.06 \mathrm{~m}$ deep crack get smoother as the interface gets shallower. This is expected because the WT has a better frequency resolution in the low-frequency range.

One can use the combined spectra or the enhanced Fourier spectrum in Fig. 9 to find the peak due to the crack. They both yield a crack frequency of $31.0 \mathrm{kHz}$, corresponding to a crack depth of $0.064 \mathrm{~m}$. Obviously, the 

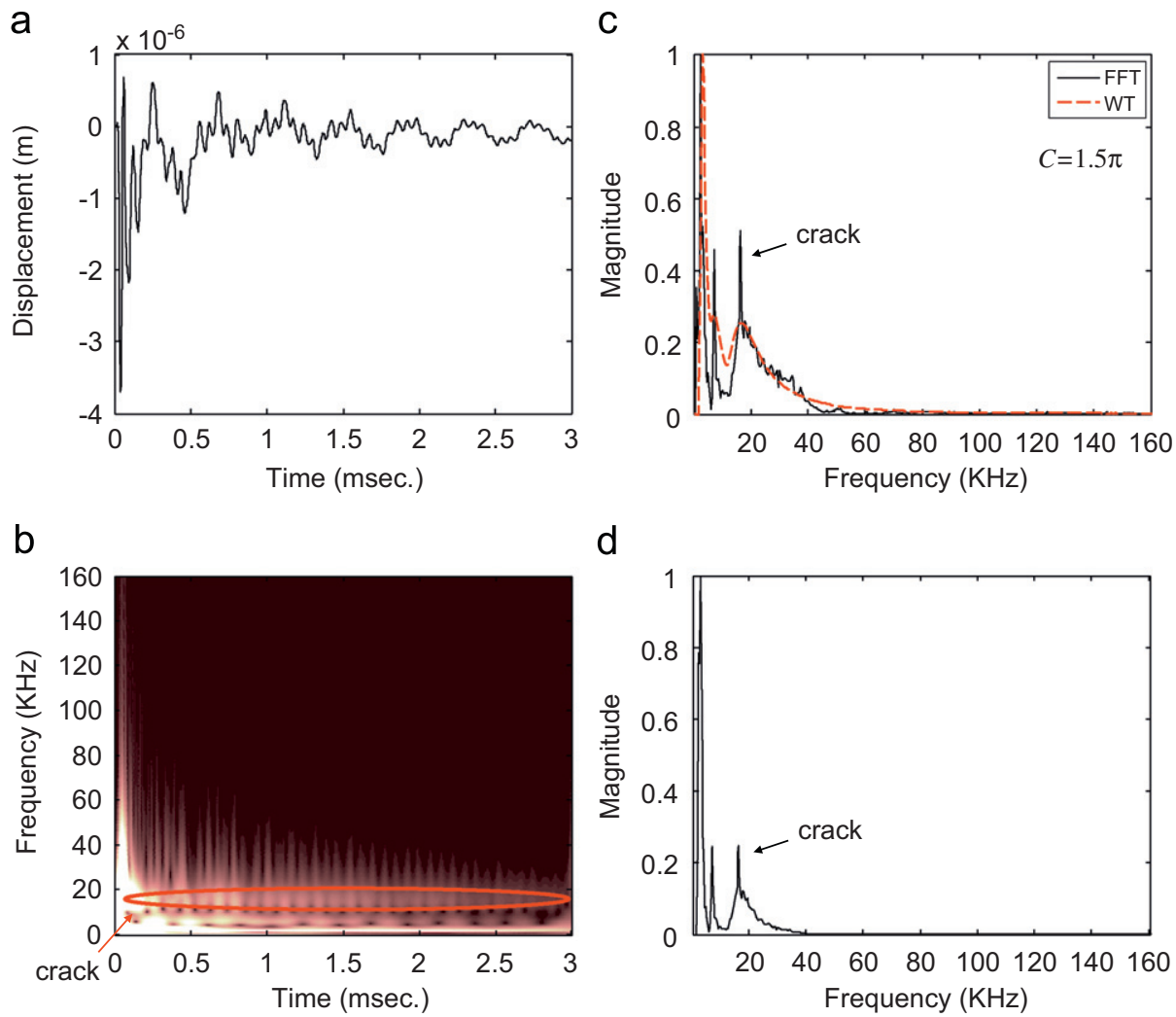

Fig. 7. Results of test 2, numerical model 1. (a) Time signal; (b) scalogram $(C=1.5 \pi)$; (c) Fourier and wavelet marginal spectra and (d) enhanced Fourier spectrum.

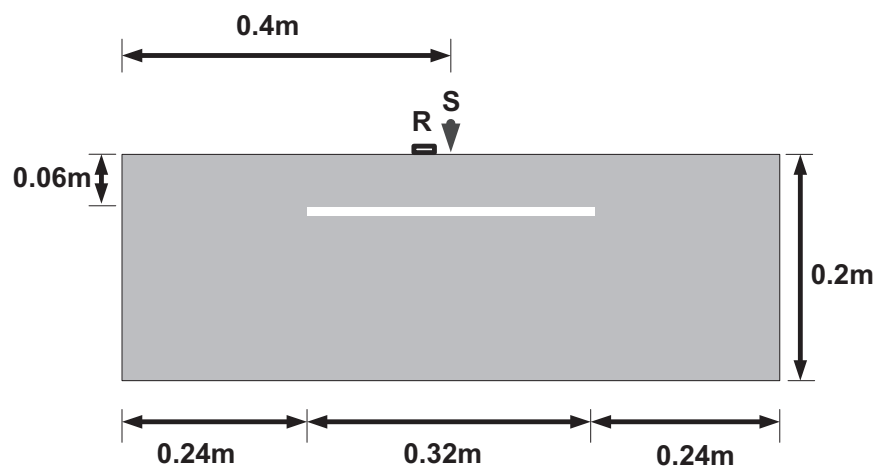

Fig. 8. Model 2.

accuracy of the crack depth is not as good as in the first case. This is expected because the reflected wave is submerged by the excessively strong vibrations. Nevertheless, the shallow crack can be detected without difficulty.

\section{Model tests}

Model tests were carried out to illustrate the signal processing methods proposed in this study. The compressive strength of the concrete is $30 \mathrm{MPa}$, the water cement ratio is 0.6 , and the concrete mix design is as follows: cement, $392 \mathrm{~kg} / \mathrm{m}^{3}$; sand, $752 \mathrm{~kg} / \mathrm{m}^{3}$; gravel, $915 \mathrm{~kg} / \mathrm{m}^{3}$; additives, $0.98 \mathrm{~kg} / \mathrm{m}^{3}$; water, $235 \mathrm{~kg} / \mathrm{m}^{3}$. The longitudinal wave velocities of models 1 and 2 are 3890 and $3900 \mathrm{~m} / \mathrm{s}$, respectively.

The experiments were conducted on two concrete specimens. The dimensions of the concrete specimens, the crack locations, and tests locations are as shown in Figs. 2 and 8 .

The measurement system is shown in Fig. 10. A steel ball with diameter $6 \mathrm{~mm}$ was dropped on the concrete specimens to produce the impact source. A conical transducer, developed at the Institute of Applied Mechanics, National Taiwan University, was adopted to measure the vertical displacement on the concrete surface. The impact and receiver locations are the same as in the numerical examples. The received voltage signals were enhanced by an amplifier and then recorded by a digital oscilloscope (LeCroy WavePro940). In the model tests, the sampling rate was $1 \mathrm{MHz}$, and the total sampling time was $5 \mathrm{~ms}$.

\subsection{Model test 1}

The time response of test 1 on model 1 is shown in Fig. 11(a). It is seen that the response curve contains a lot of noise. For the first few cycles of fluctuation, the experimental response resembles the numerical curve in Fig. 3. However, the experimental response does not diminish quickly. Instead, vibration seems to arise. Such vibration is not seen in the numerical curve because 
a

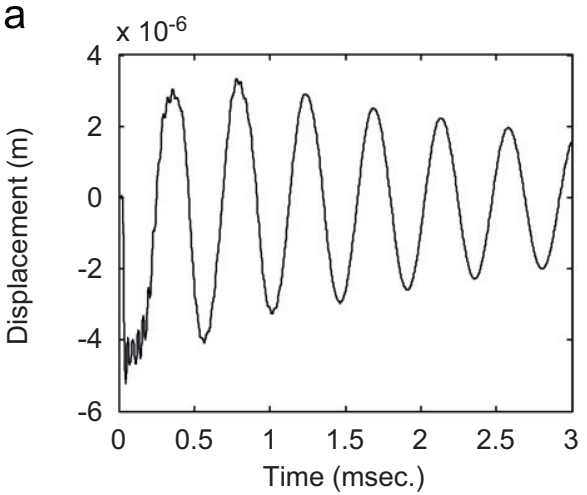

b

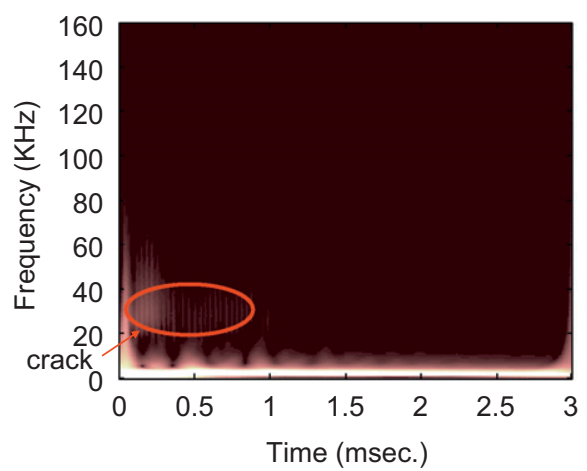

C

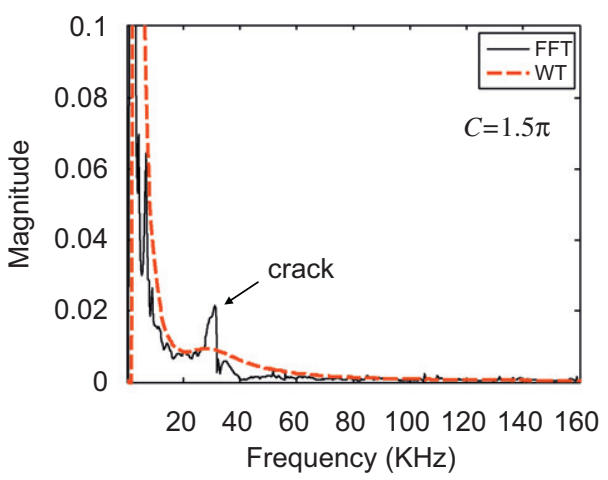

d

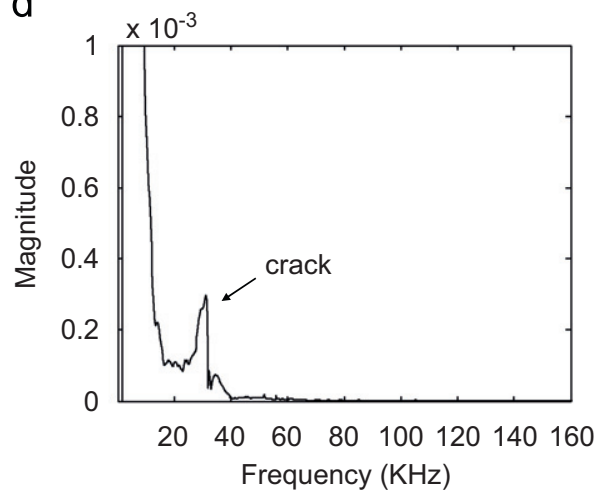

Fig. 9. Results of numerical model 2. (a) Time signal; (b) scalogram $(C=1.5 \pi)$; (c) Fourier and wavelet marginal spectra and (d) enhanced Fourier spectrum.

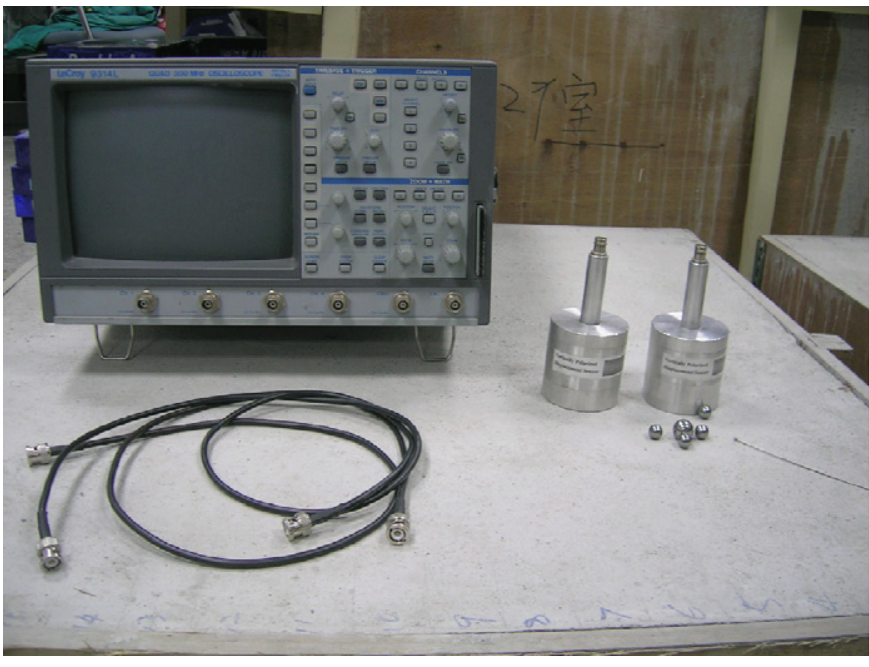

Fig. 10. The experimental setup.

absorbing boundary conditions were applied on the sides of the numerical models.

Fig. 11(b) shows the scalogram of test 1. One can find a bright vertical stripe caused by the strong surface wave near the origin. There is also a bright band at about $10 \mathrm{kHz}$ caused by the bottom echo. There are two bright bands at the bottom of the experimental scalogram. Compared with the result of modal analysis, these bands correspond to two vibration modes with frequencies 2.46 and $3.63 \mathrm{kHz}$.
Fig. 11(c) shows the Fourier spectrum and the wavelet marginal spectrum of test 1 . The difference between the Fourier spectrum and the wavelet marginal spectrum becomes more evident in the model test. The Fourier spectrum contains a lot of interferences, part from the transform and part from signal noise. The wavelet marginal spectrum, on the other hand, is very smooth. There is only a hump in the spectrum other than the vibration peaks. Although the hump is rounded, it provides a clue that the peak should occur somewhere around $10 \mathrm{kHz}$. Then, one can resort to the Fourier spectrum to decide the location of the peak easily. One can also pinpoint the peak by using the enhanced Fourier spectrum in Fig. 11(d) directly. The frequency of the peak is $10.3 \mathrm{kHz}$, which yields a bottom depth of $0.204 \mathrm{~m}$.

The second test was performed on the top of the $0.12 \mathrm{~m}$ deep horizontal crack. The response curve and the scalogram are shown in Fig. 12(a) and (b), respectively. The response curve exhibits the same attributes as in test 1 . The scalogram is not as neat as in the numerical examples. Yet, the bright bands for the surface wave, the crack echo, and the modal vibrations can be identified easily.

Fig. 12(c) and (d) show the Fourier spectrum, the wavelet marginal spectrum and the enhanced Fourier spectrum of test 2. The characteristics of these spectra are the same as in test 1. Although the Fourier spectrum contains noise, one can apply the combined spectra or the enhanced Fourier spectrum to identify the crack peak 

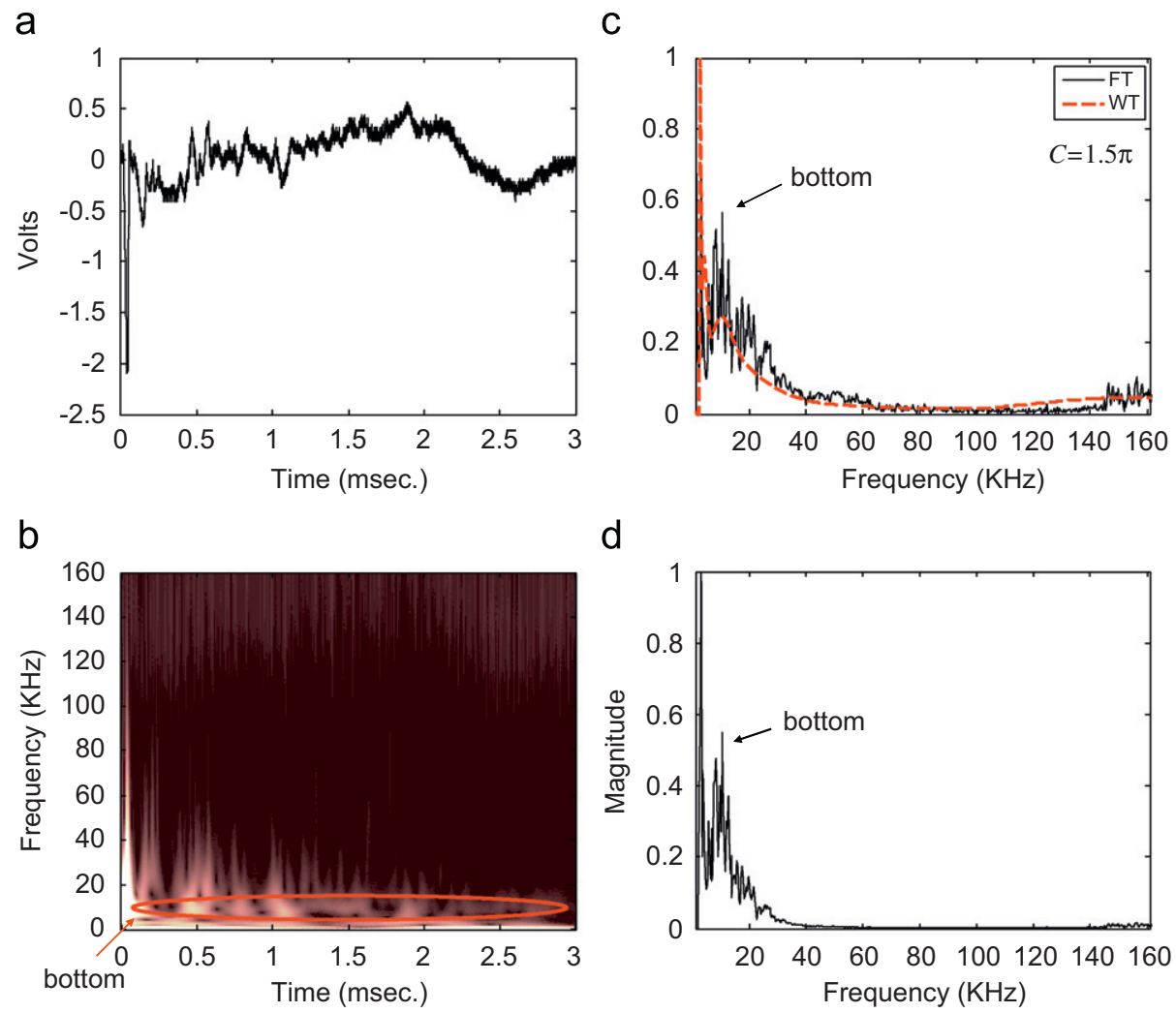

Fig. 11. Results of test 1, experimental model 1. (a) Time signal; (b) scalogram ( $C=1.5 \pi)$; (c) Fourier and wavelet marginal spectra and (d) Fourier and wavelet marginal spectra.

a

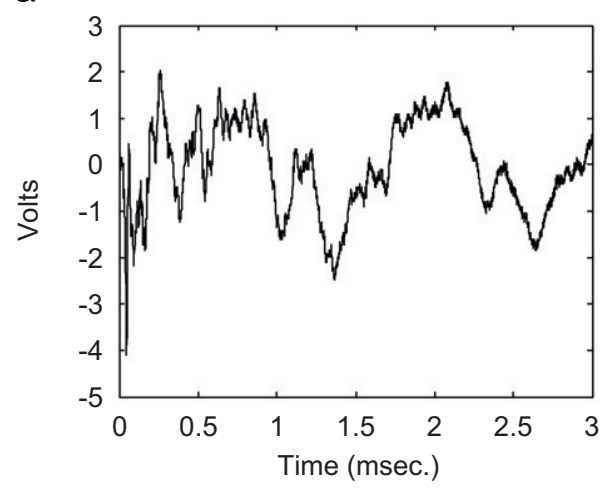

b

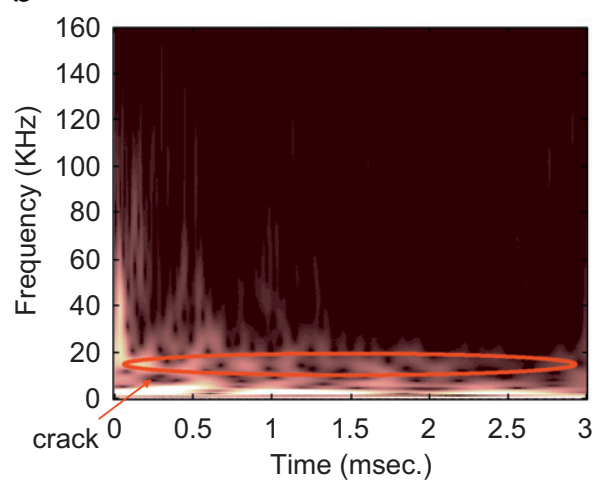

C

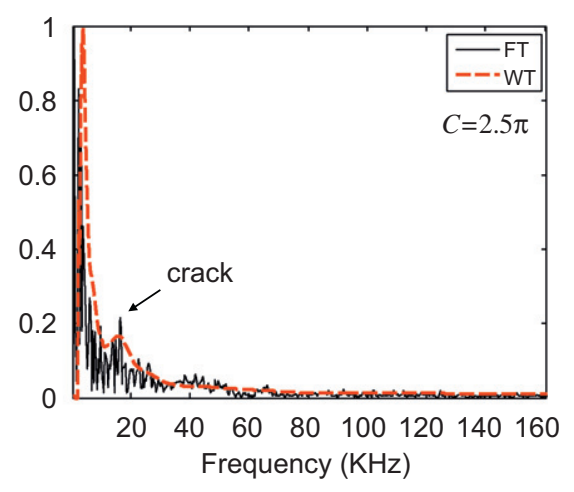

d

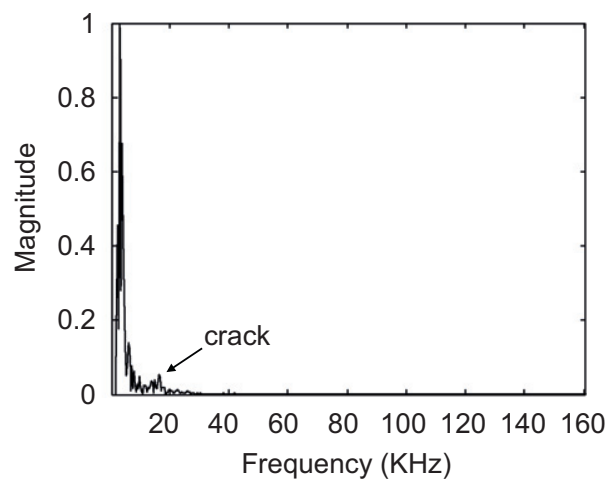

Fig. 12. Results of test 2, experimental model 1. (a) Time signal; (b) scalogram $(C=2.5 \pi)$; (c) Fourier and wavelet marginal spectra and (d) enhanced Fourier spectrum. 
a

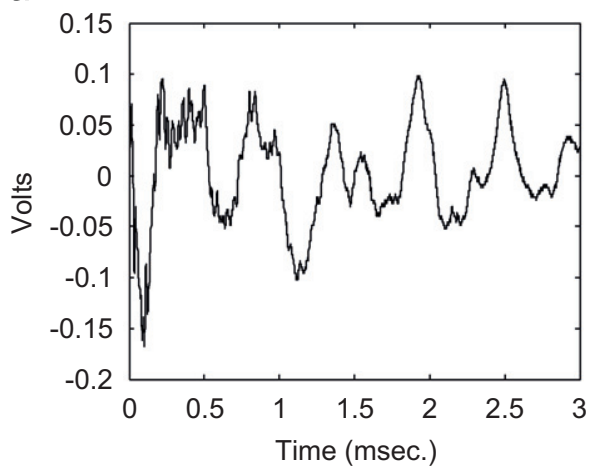

b

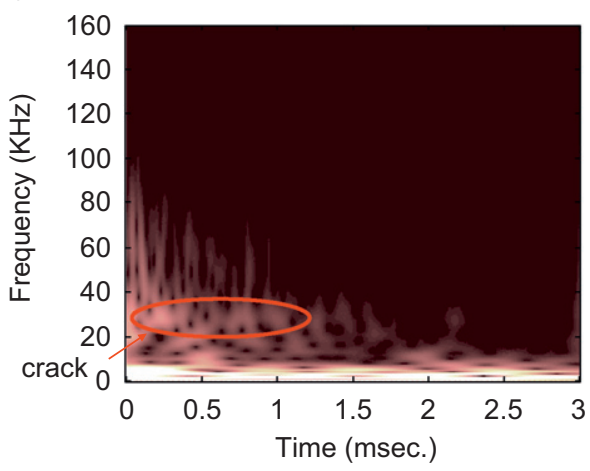

C

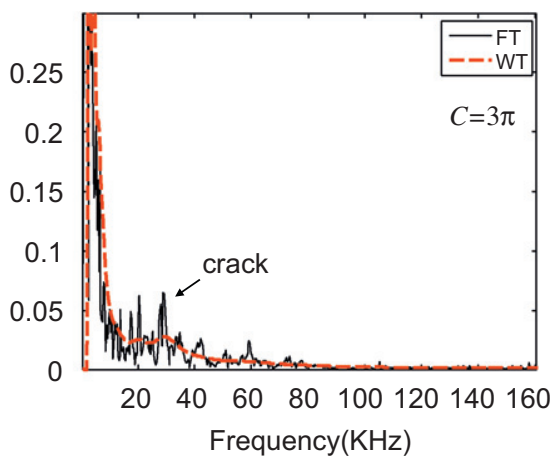

d

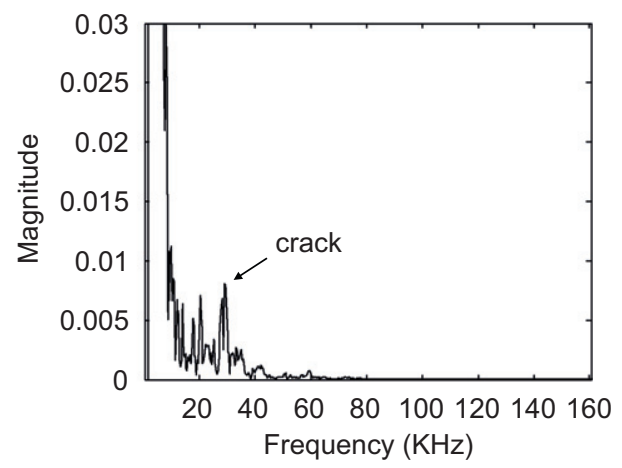

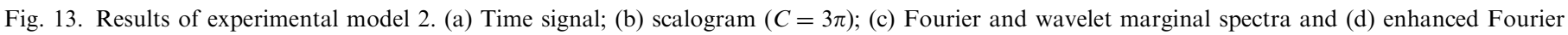
spectrum.

easily. The frequency of the peak is $16.7 \mathrm{kHz}$, which yields a bottom depth of $0.115 \mathrm{~m}$.

\subsection{Experimental model 2}

In the second experimental model, the crack is $0.06 \mathrm{~m}$ beneath the impact point, as shown in Fig. 8.

The results of this model test are shown in Fig. 13. The brightest band on the bottom of scalograms comes from the high-energy flexural vibration of the plate above crack. This was verified by a modal analysis, and there is indeed a flexural vibration mode at $2.46 \mathrm{kHz}$. Although the energy of crack echo is much smaller than the flexural vibration, a bright band is still observed at about $30 \mathrm{kHz}$.

Examining the Fourier spectrum and the wavelet marginal spectrum in Fig. 13(c), one can see that the situation is even worse than in model test 1 . There are many peaks in the Fourier spectrum, and the true crack peak is extremely difficult to identify. This is because the energy of the crack echo is so small that any interference may form peak of the same magnitude. Fortunately, the wavelet marginal spectrum only contains a single hump other than the peak of flexural vibration. Although the hump is very smooth, it reveals that the crack peak is located at around $30 \mathrm{kHz}$. Hence, one can apply the Fourier spectrum to find the location of the peak. The frequency of the peak is $33.0 \mathrm{kHz}$, and the depth of the crack is $0.059 \mathrm{~m}$.
The enhanced Fourier spectrum in Fig. 13(d) can also be used to find the crack peak. However, it still contains a lot of noise. Hence, it is advisable to take the wavelet marginal spectrum into account when dealing with such difficult situation.

\section{Conclusion}

This paper applies the WT in the analysis of the response signal received in the impact echo test. The WT was first compared with the conventional FT theoretically. Then, numerical simulation and model tests were conducted to check the performance of these transforms.

From the numerical examples and model tests, it is seen that surface wave, ripples, and multiple peaks complicate the Fourier spectrum and jeopardize the diagnosis of structures. The WT, on the other hand, is free from such interference and provides more information than the Fourier spectrum.

The surface wave, the echo signals, and vibration signals form bright bands in the WT scalogram. Based on the sizes and locations of the bright bands, one may estimate the duration and frequency content of those waves.

The wavelet marginal spectrum is similar to the Fourier spectrum, but it contains no ripples and multiple peaks. Hence, it is easier to identity the echo peak in the marginal spectrum than in the Fourier spectrum, especially for the experimental data. The frequency resolution of the wavelet 
marginal spectrum is not as good as the Fourier spectrum, however.

Two approaches are proposed in this paper to overcome the drawback. One is to combine the Fourier spectrum and the wavelet marginal spectrum in the same figure. The wavelet marginal spectrum is firstly examined to find the correct echo peak. Then, in the vicinity of the WT peak, the Fourier spectrum is used to determine the precise location of the echo peak. The second approach is to take the product of the Fourier spectrum and the wavelet marginal spectrum to obtain the enhanced Fourier spectrum. As such, the interference in the Fourier spectrum is suppressed while the peak is enhanced. Both approaches were proved to be effective in the detection of deep and shallow cracks. Hence, they provide useful tools in the analysis of impact echo data.

\section{Acknowledgment}

This work was supported by the National Science Council, Republic of China under grant NSC 90-2211-E002-079.

\section{References}

[1] Sansalone M, Carino NJ. Impact-echo: a method for flaw detection in concrete using transient stress waves. NBSIR 86-3452. Gaithersburg, MD: National Bureau of Standards, September 1986. p. 222.

[2] Lin Y, Sansalone M, Carino NJ. Finite element studies of the transient response of plates containing thin layers and voids. J Nondestruct Eval 1990;9:27-47.

[3] Lin Y, Sansalone M. Transient response of thick and square bars subjected to transverse elastic impact. J Acoust Soc Am 1992;91: $885-93$.

[4] Cheng C, Sansalone M. The impact-echo response of concrete plates containing delaminations: numerical, experimental and field studies. Mater Struct 1993;26:274-85.

[5] Liang MT, Su PJ. Detection of the corrosion damage of rebar in concrete using impact-echo method. Cement Concrete Res 2001;31: 1427-36.

[6] Abraham O, Leonard C, Cote P, Piwakowski B. Time-frequency analysis of impact-echo signals: numerical modeling and experimental validation. ACI Mater J 2000;97:645-57.

[7] Kim YY, Kim EH. Effectiveness of the continuous wavelet transform in the analysis of some dispersive elastic waves. J Acoust Soc Am 2001;110:86-94.
[8] Chiang $\mathrm{CH}$, Cheng CC. Detecting rebars and tubes inside concrete slabs using continuous wavelet transform of elastic waves. J Mech 2004;20:297-302.

[9] Shokouhi P, Gucunski N, Maher A. Time-frequency techniques for the impact-echo data analysis and interpretations. In: Proceedings of the 9th European conference on non-destructive testing, ECNDT, Berlin, 2006. p. 1-10.

[10] Algernon D, Wiggenhauser H. Anwendung der hilbert-huang transformation zur auswertung von impact-echo datensdtzen. In: Proceedings of German society for non-destructive testing annual convention (DGZfP Jahrestagung), Rostock, 2005. p. 1-13.

[11] Leonard F. Phase spectrogram and frequency spectrogram as new diagnostic tools. Mech Syst Signal Process 2007;21:125-37.

[12] Jeong H, Jang YS. Fracture source location in thin plates using the wavelet transform of dispersive waves. IEEE Trans Ultrason 2000;47:612-9.

[13] Obaidat MS, Suhail MA, Sadoun B. An intelligent simulation methodology to characterize in materials. Inform Sci 2001;137: $33-41$.

[14] Yan YJ, Yam LH. Detection of delamination damage in composite plates using energy spectrum of structure dynamic responses decomposed by wavelet analysis. Comput Struct 2003;82:347-58

[15] Cook DA, Berthelot YH. Detection of small surface-breaking fatigue cracks in steel using scattering of Rayleigh waves. NDT E Int 2001;34:483-92.

[16] Darowicki K, Zakowski K. A new time-frequency detection method of stray current field interference on metal structures. Corros Sci 2004;46:1061-70.

[17] Simpson WA. Time-frequency-domain formulation of ultrasonic frequency analysis. J Acoust Soc Am 1974;56:1776-81.

[18] Cohen L. Time-frequency analysis. New Jersey: Prentice-Hall; 1995.

[19] Yeh PL. The time-frequency domain analysis and image method of the impact echo method. Taipei: National Taiwan University; 2006.

[20] Mallat S. A wavelet tour of single processing. 2nd ed. New York: Academic Press; 1999.

[21] Daubechies I. Ten lectures on wavelets. Philadelphia, PA: Society for Industrial and Applied Mathematics; 1992.

[22] Stanislaw RM. Wavelet analysis for processing of ocean surface wave records. Ocean Eng 2001;28:957-87.

[23] Misiti M, Misiti Y, Oppenheim G, Poggi JM. Wavelet toolbox 4 user's guide. The Math Works, Inc.; 2007.

[24] Hallquist JO. LS-DYNA keyword user's manual. Livermore: Livermore Software Technology Corporation; 2003.

[25] Goldsmith W. Impact: the theory and physical behavior of colliding solids. London: Edward Arnold Ltd.; 1965.

[26] Colla C, Lausch R. Influence of source frequency on impact-echo data quality for testing concrete structure. NDT\&E Int 2003;36: 203-13. 